\title{
Preface: Eli Sercarz Memorial Issue, Part I
}

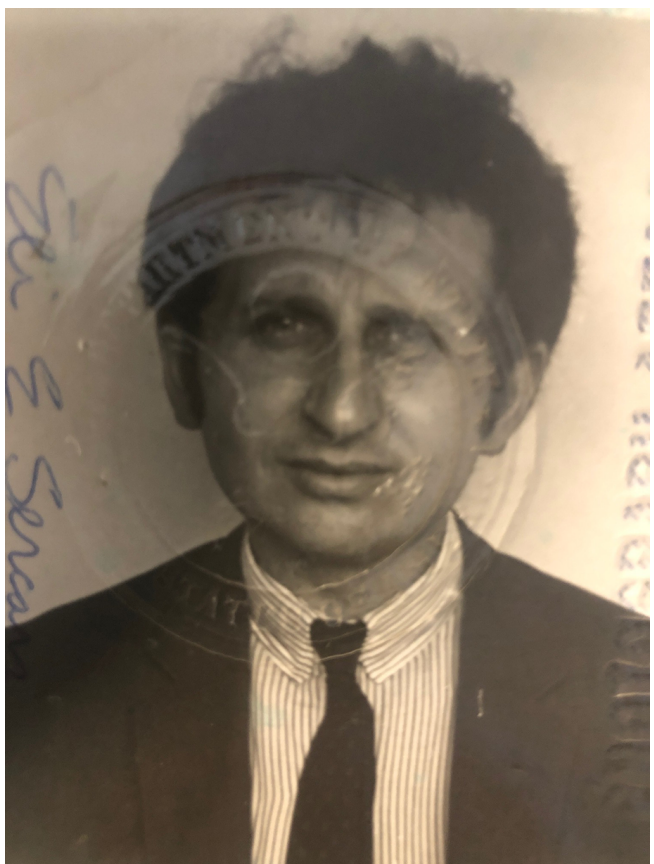

The late Eli E. Sercarz devoted his life and career to the field of immunology, and he systematically dissected several of the unknown fundamental principles and molecular mechanisms that govern the complex immune response mediated by immune cells (T, B, APC). His research investigations and approaches were novel and creative, delineating the means by which specific amino acid sequences of protein molecules dictate a response (immunogenic) or nonresponsiveness (tolerogen) via sophisticated interactions among the APC, T lymphocyte subsets, and B cells. Such meticulous analyses using various protein molecules as models resulted in clarifications of the observed immune defects involved in various human diseases (autoimmune, transplantation, etc.). Eli's guidance and traineeships emanated in hundreds of publications and nurtured the education and development of a large number of academic immunologists, whose careers and research investigations were the direct extensions of his fundamental research.
Eli died at the age of 75 after battling renal cancer. This is the first publication celebrating his memory and legacy. Vipin Kumar and Benjamin Bonavida, guest editors for these special issues in Critical Reviews $^{\mathrm{TM}}$ in Immunology (CRI), were previously Eli's fellows and decided to dedicate three issues in his memory. As anticipated, many of Eli's former fellows and collaborators have responded enthusiastically about dedicating their work in these three issues of CRI, published simultaneously in his memory.

In the first CRI issue, there are eight contributions, outlined briefly as follows:

Luis Soares et al.'s article entitled "A Common Druggable Defect in Regulatory T Cells from Patients with Autoimmunity" discusses the role and function of the regulatory $\mathrm{T}$ cells (Tregs) in autoimmune diseases. Soares and colleagues examined the Tregs cells isolated from the blood of patients with T1D and SLE, who were treated with low concentrations of IL-2 and compared to control individuals. They discovered a defect in the IL2R-mediated signaling pathway in the patients' Tregs, which led to the JAK1 of STAT5 activation, required for the function of Tregs. This results from the degradation of the IL-2R $\beta$ chain and loss of pSTAT5 expression. Interestingly, they were able to restore the function of the Tregs in in vivo murine models for SLE and T1D with an inhibitor of neddylation - a drug known as "neddylation activating enzyme inhibitor" (NAE1). This review discusses the various molecular pathways that resulted from their discovery.

Irun Cohen et al.'s article is entitled "Immune Computation and COVID-19 Mortality: A Rationale for IVIg." It has been reported that COVID-19 infection is more lethal in older persons than in the young, due to an overactive inflammatory response. Cohen and colleagues describe the differences and similarities between the immune regulation of the inflammatory response phenotype and machine learning algorithms used with computers. They speculated that a response to COVID-19 infection might be induced by treating the elderly patients 
with a wellness repertoire of antibodies obtained from healthy young people and administered in the form of IVIg.

Stopforth and Ward's article, entitled "The Role of Antigen Presentation in Tumor-Associated Macrophages," discusses the multiple roles of tumor-associated macrophages (TAMs) that are present in the tumor microenvironment (TME) and that have been reported with contrasting functions, although they typically have been considered to be of the antitumor phenotypes and, hence, strategies have been devised to target them. The TAMs are not as efficient as the APCs in antigen-processing and antigen-presentation, and their role in the TME is discussed with the goal of designing novel immunotherapies that will enhance their antitumor activities.

Lehmann and Lehmann's article entitled "Aleatory Epitope Recognition Prevails in Human $\mathrm{T}$ Cell Responses?" discusses the interesting notion of each individual's immune response to only a fraction of potential epitopes based on the MHC alleles present in that particular individual, along with the expressed epitope space, which appears to be a random process. In the murine models analyzed, Lehmann and Lehmann have found that the preselection of actually recognized determinants was erratic. This confirms Eli's hypothesis and findings regarding the phenomenon he dubbed "aleatory determinant recognition" (with alea meaning "dice" in Latin).

Gabaglia et al.'s article entitled "The Potential for Immunospecific Therapy in Multiple Sclerosis Based on Identification of Driver Clones of the Disease" reviews their proposed mechanism of the $T$ cell subpopulation responsible for the induction and relapse of patients with MS. Gabaglia and collaborators have focused on the concept of a "driver clone," as noted in their investigations in mice with EAE. In contrast to prior findings, they propose that the driver $\mathrm{T}$ cells are the same for induction as well as relapse. Accordingly, their findings open new personalized immunotherapeutic strategies for MS patients.

Di Sante et al.'s article entitled "Past and Future of the Molecular Characterization of the $T$ Cell Repertoire: Some Highlights of Eli Sercarz's" reviews Eli's initial research on the spectratype that contributes to the underlying molecular characterization of the $\mathrm{T}$ cell repertoire through immunoscope analysis. Such studies were fundamental in gaining increased insight into the immunopathogenesis of autoimmmune diseases, infections, and cancer.

Zanetti's article entitled "Ubi Maior Minor Cessat" is a testament to Eli's vision related to the hierarchical order of the $\mathrm{T}$ cell epitopes that are used for antigen presentation and $\mathrm{T}$ cell activation. In this review, Zanetti reviews the concepts of epitope dominance and crypticity that determine $\mathrm{T}$ cell-cell cooperation and result in an adaptive immune response. In addition, this review reiterates the mechanisms underlying tolerance to self-antigens and how cryptic $\mathrm{T}$ cell epitopes may generate antitumor immune responses.

Kumar's article entitled "A Coordinated Dance of the Effector and Regulatory T Cells Reactive to Different Determinants in the Orchestration of an Autoimmune Response" reviews many of the complex immune cell-cell interactions in the regulation of experimental autoimmune encephalomyelitis (EAE), a prototype autoimmune disease for multiple sclerosis. Vipin reviews original studies that originated in Eli's laboratory and his subsequent studies that expanded into new concepts and discoveries and novel mechanisms related to Treg activation and their pivotal role in the maintenance of immune homeostasis. Elaborate reviews on the regulatory $\mathrm{T}$ cell subsets $\left(\mathrm{CD}^{+}\right.$and $\left.\mathrm{CD} 8^{+}\right)$offer insights on both the innate and adaptive mechanisms of immune regulation. Kumar reviews several facets of immune regulation and epitope selectivity and specificity during an immune response. Some examples of the topics covered herein are (a) immune responses spreading to subdominant and/or cryptic determinants; (b) MHC-binding affinity versus Th1/Th2 balance; (c) the oligoclonality of $\mathrm{T}$ cells reactive to a dominant determinant; (d) deletion of the naturally induced regulatory $\mathrm{CD} 4^{+} \mathrm{T}$ cells, which leads to chronic disease; and (e) the discovery of innate-like and unconventional $\mathrm{PLZF}^{+} \mathrm{CD} 8 \alpha \alpha^{+} \mathrm{T}-$ $\mathrm{CR} \alpha \beta^{+}$regulatory CD8 T cells in both mice and humans. These topics emphasize the importance 
and implications of novel interventions for autoimmune diseases.

The provided reviews discuss up-to-date studies on various immune system topics, several of which resulted from expansions of original findings from Eli's laboratory. The contents of this CRI issue also emphasize the many outcomes of Eli's significant conceptual contributions for managing human diseases today.
Guest Editors:

Vipin Kumar

University of California San Diego

La Jolla, CA, USA

Benjamin Bonavida

University of California, Los Angeles

Los Angeles, CA, USA 